\title{
Quantum Associative Memory with Exponential Capacity
}

\author{
Dan Ventura and Tony Martinez \\ Neural Network and Machine Learning Laboratory (http://axon.cs.byu.edu) \\ Department of Computer Science, Brigham Young University, Provo, UT 84602
}

\begin{abstract}
Quantum computation uses microscopic quantum level effects to perform computational tasks and has produced results that in some cases are exponentially faster than their classical counterparts by taking advantage of quantum parallelism. The unique characteristics of quantum theory may also be used to create a quantum associative memory with a capacity exponential in the number of neurons. This paper covers necessary high-level quantum mechanical ideas and introduces a simple quantum associative memory. Further, it provides discussion, empirical results and directions for future work.
\end{abstract}

\section{Introduction}

We consider the problem of associative pattern completion -- given a set of patterns of the form $x_{i} \rightarrow o_{i}$, learn to produce the vector $o_{i}$ given the vector $x_{i}$. The trivial solution is simply to store the set of patterns as a lookup table. The problem becomes interesting with the added constraint of finding as small a representation as possible. This paper proposes a quantum associative memory (QuAM) with a storage capacity of $O\left(2^{n}\right)$, while using only $n$ neurons.

The field of quantum computation, which applies ideas from quantum mechanics to the study of computation, was introduced in the mid 1980's [6]. For a readable introduction to quantum computation see [3]; for a more rigorous treatment see for example [1]. The field is still in its infancy and very theoretical but offers exciting possibilities for the field of computer science -- the most notable to date being the discovery of quantum computational algorithms for computing discrete logarithms and prime factorization in polynomial time, two problems for which no known classical polynomial time solutions exist [11]. These algorithms provide theoretical proof not only that interesting computation can be performed at the quantum level but also that it may in some cases have distinct advantages over its classical cousin. The quantum computing approach to solving these two problems takes advantage of the unique features of quantum systems to process an exponential number of possibilities simultaneously, thus providing exponential speedup over classical approaches.

This paper presents a unique reformulation of the pattern classification problem into the language of wave functions and operators. This reformulation may be generalized to a very large class of computational learning problems, opening up the possibility of employing the capabilities of quantum computational systems for the solution of computational learning problems. Here we examine one such problem, that of pattern completion, and show how it may be handled in the new formalism. Further, it is shown that the possibility of utilizing quantum effects such as coherence and interference (see section 2) may have great advantage over classical computational learning methods.

Artificial neural networks (ANN) seek to provide ways for classical computers to learn rather than to be programmed. If quantum computers become a reality, then artificial neural network methods that are amenable to and take advantage of quantum mechanical properties will become possible. Further, the possibility of processing an exponential space in polynomial time is very appealing. In particular, can quantum mechanical properties be applied to ANNs for problems such as associative memory? Recently, some work has been done in the area of combining classical artificial neural networks with ideas from the field of quantum mechanics [2], [8], [10], [12]. This paper introduces some important ideas from quantum mechanics, proposes a quantum associative memory, presents analytical discussion, and produces empirical results that support the analysis.

\section{Quantum mechanics}

Quantum mechanics is a theory that in many ways is counterintuitive. Yet it has provided us with perhaps the most accurate physical theory (in terms of predicting experimental results) ever devised by science. The theory is well-established and is covered in its basic form by many textbooks (see for example [7]). Several necessary ideas from this theory are briefly reviewed here. 
Linear superposition is closely related to the familiar mathematical principle of linear combination of vectors. Quantum systems are described by a wave function $\psi$ that exists in a Hilbert space. In the Hilbert space there exists a set of states, $\left|\phi_{i}\right\rangle$, that form a basis, and the system is described by a quantum state $|\psi\rangle$,

$$
|\psi\rangle=\sum_{i} c_{i}\left|\phi_{i}\right\rangle .
$$

$|\psi\rangle$ is said to be in a linear superposition of the basis states $\left|\phi_{i}\right\rangle$, and in the general case, the coefficients $c_{i}$ may be complex. We use here the Dirac bracket notation where the ket $|\cdot\rangle$ is analogous to a column vector, and the bra $\langle\cdot|$ is analogous to the complex conjugate transpose of the ket. In quantum mechanics the Hilbert space and its basis have a physical interpretation, and this leads directly to perhaps the most counterintuitive aspect of the theory. The counter intuition is this -- at the microscopic or quantum level, the state of the system is described by the wave function $\psi$, that is, as a linear superposition of all basis states. However, at the macroscopic or classical level the system can be in only a single basis state. For example, at the quantum level an electron can be in a superposition of many energy levels; however, in the classical realm this obviously cannot be.

Coherence and decoherence are closely related to the idea of linear superposition. A quantum system is said to be coherent if it is in a linear superposition of its basis states. A strange result of quantum mechanics is that if a system that is in a linear superposition of states interacts in any way with its environment, the superposition is destroyed. This loss of coherence is called decoherence and is governed by the wave function $\psi$. The coefficients $c_{i}$ are called probability amplitudes, and $\left|c_{i}\right|^{2}$ gives the probability of $|\psi\rangle$ collapsing into state $\left|\phi_{i}\right\rangle$ if it decoheres. In the Dirac notation, the probability that a quantum state $\left|\psi_{2}\right\rangle$ will collapse into an eigenstate $\left|\phi_{i}\right\rangle$ is written $\left|\left\langle\phi_{i} \mid \psi\right\rangle\right|^{2}$ and is analogous to the dot product (projection) of two vectors. Consider, for example, a discrete physical variable called spin. The simplest spin system is a two-state system, called a spin-1/2 system, whose basis states are usually represented as $|\uparrow\rangle$ (spin up) and $|\downarrow\rangle$ (spin down). In this simple system the wave function $\psi$ is a distribution over two values (up and down) and a coherent state $|\psi\rangle$ is a linear superposition of $|\uparrow\rangle$ and $|\downarrow\rangle$. One such state might be

$$
|\psi\rangle=\frac{2}{\sqrt{5}}|\uparrow\rangle+\frac{1}{\sqrt{5}}|\downarrow\rangle .
$$

As long as the system maintains its quantum coherence it cannot be said to be either spin up or spin down. It is in some sense both at once. Classically, of course, it must be one or the other, and when this system decoheres the result is, for example, the $|\uparrow\rangle$ state with probability

$$
|\langle\uparrow \mid \psi\rangle|^{2}=\left(\frac{2}{\sqrt{5}}\right)^{2}=.8 .
$$

Operators on a Hilbert space describe how one wave function is changed into another. Here they will be denoted by a capital letter with a hat, such as $\hat{A}$, and they may be represented as matrices acting on vectors. Using operators, an eigenvalue equation can be written

$$
\hat{A}\left|\phi_{i}\right\rangle=a_{i}\left|\phi_{i}\right\rangle,
$$

where $a_{i}$ is the eigenvalue. The solutions $\left|\phi_{i}\right\rangle$ to such an equation are called eigenstates and can be used to construct the basis of a Hilbert space as discussed above. In the quantum formalism, all properties are represented as operators whose eigenstates are the basis for the Hilbert space associated with that property and whose eigenvalues are the quantum allowed values for that property. It is important to note that operators in quantum mechanics must be linear operators.

Interference is a familiar wave phenomenon. Wave peaks that are in phase interfere constructively (magnify each other's amplitude) while those that are out of phase interfere destructively (decrease or eliminate each other's amplitude). This is a phenomenon common to all kinds of wave mechanics from common water waves to optics. The well-known double slit experiment demonstrates empirically that at the quantum level interference also applies to the probability waves of quantum mechanics. Herein lies a truly non-classical property that gives computation by quantum means a unique advantage over classical approaches, including any stochastic methods [5].

Entanglement is the potential for quantum states to exhibit correlations that cannot be accounted for classically. Mathematically, states that cannot be factorized (as a tensor product) are said to be entangled, while those that can be factorized are not. There are different degrees of entanglement, and it is interesting to note from a computational standpoint that quantum states that are superpositions of only those basis states that are maximally far apart in terms of Hamming distance are those states with the greatest entanglement. For example, a superposition of only the states $|00\rangle$ and $|11\rangle$ is maximally entangled; on the other hand, a superposition of $|00\rangle,|01\rangle$ and $|11\rangle$ still exhibits entanglement, but to a lesser degree. Finally, it should be mentioned that while interference is a quantum property that has a classical cousin, entanglement is a completely quantum phenomenon for which there is no classical analog.

\section{Quantum associative memory (QuAM)}

The quantum associative memory proposed here consists of a set of spin systems, each of which may be thought of as the quantum analog of a simple 2 or 3 state artificial neuron. The patterns to be learned are viewed as 
operators that act on the spin systems. The following definitions are necessary for describing the QuAM:

$\mathrm{r}$

$x$ as a binary input vector of length $n$, whose elements can have the values 0 or 1 ,

$z$ as a ternary feature vector of length $n$, whose elements can have the values 0,1 , or *, with * $r$ representing a "don't care" state,

$o$ as a binary output class vector of length $s$ associated $r$ with an input vector $x$,

$p$ as a binary output class vector of length $s$ associated with a feature vector $z$, and

$T$ as a set of patterns $x_{i} \rightarrow o_{i}, 1 \leq \mathrm{i} \leq q$ to be learned.

A feature vector $\underset{z}{z}$ is said to match an input vector $\stackrel{r}{x}$ if and only if $\forall j, z_{j}=x_{j}$ or $z_{j}=*$. Further, if the number of elements $z_{j} \neq *$ is $r$, then the feature vector $z$ is of order $r$, or $z$ is said to be an $r$ th order feature. For example, given the input vector (101), the 1 st order features that match it are $(1 * *),(* 0 *)$, and $(* * 1)$. Additional necessary definitions include the matching functions $m$ and $w$. Together these two functions compute a weighted hamming score for how closely a feature vector matches an input vector.

$$
\begin{aligned}
& m(x, z)= \begin{cases}1 & \text { if } z=* \\
\rho & \text { if } x=z \\
0 & \text { otherwise. }\end{cases} \\
& w\left(\begin{array}{r}
r \\
\underset{r}{r}
\end{array}\right)= \begin{cases}0 & \text { if } \exists j, m\left(x_{j}, z_{j}\right)=0 \\
\prod_{j} m\left(x_{j}, z_{j}\right) & \text { otherwise. }\end{cases}
\end{aligned}
$$

For computational purposes, the most commonly used quantum system is the spin-1/2 system introduced in Section 2, which is referred to as a quantum bit or qubit. Relabeling the eigenstates as $|0\rangle$ and $|1\rangle$, it is easy to see why this is so. One useful feature of quantum computation is that as long as a set of $n$ qubits maintains its quantum coherence, it can be considered to be all $2^{n}$ possible binary patterns at once. Thus any operation performed on the qubits is performed on all $2^{n}$ patterns in parallel. This is termed quantum parallelism.

We propose the use of a slightly more complex spin-1 system (3 states) whose eigenstates are labeled $|0\rangle,|1\rangle$ and $|*\rangle$. This system may be thought of as a classical ternary variable --- when it decohers it will have a value of either 0 or 1 or $*$. However, while coherence is maintained, it will exist as a superposition of all three. Thus, a coherent set of $n$ such quantum systems would be represented as a superposition of all $3^{n}$ eigenstates. We now define the quantum system for learning associative pattern completion: $|\psi\rangle=\sum_{z p}^{\mathrm{rr}} c_{z p}^{\mathrm{rr}}|\underset{z p}{\mathrm{rr}}\rangle$ as the quantum state of a set of $n$ spin-1 systems and $s$ spin-1/2 systems, with the former designated to represent the input vector and the latter designated to represent the output class vector.

Each spin-1 system can be thought of as a neuron. Notice that the eigenstates are labeled as a feature vector $(z)$ paired with an output vector $(p)$, corresponding to the different states in which the quantum system can be found. Each basis state corresponds to a possible pattern or partial pattern, and a superposition of states corresponds to a superposition of different patterns and partial patterns. Therefore, $\psi$ represents a QuAM with the ability to represent $3^{n} \times 2^{s}$ different patterns. In this sense it is no different than a classical pattern associator. As we shall see, however, its storage capacity will be exponential in $n$. The patterns to be learned are considered as operators that operate on $\psi$, with each pattern producing two operators defined as follows. Consider the operators as matrices that are indexed by column and row as $z_{1} p_{1}, z_{2} p_{2}$. Then the definition for the first set of operators, $A_{x_{i} o_{i}}^{r}$, is

$$
a_{z_{1} p_{1}, z_{2} p_{2}}^{r r}= \begin{cases}0 & \text { if } \underset{r}{r} \text { does not match } x_{i} \\ w\left(x_{i}, z_{1}\right) & \text { if } z_{1}=z_{2} \text { and } o_{i}=p_{1}=p_{2} .\end{cases}
$$

The $\hat{A}$ operators are designed to increase the probability amplitudes of those eigenstates in $|\psi\rangle$ associated with features that match the input vectors in the training set. An $\hat{A}$ operator increases the probability amplitude for only those eigenstates whose features match its associated input training vector and whose output class matches the output training class. The second set of operators, $B_{x_{i} o_{i}}^{\mathrm{r}}$, is defined as

$$
b_{z_{1} p_{1}, z_{2} p_{2}}^{r} \underset{r}{r}= \begin{cases}0 & \text { if } p_{1}^{r} \text { does not match } x_{i} \\ -w_{i}\left(x_{i r} z_{1}\right) & \text { if } z_{1}=z_{2} \text { and } q_{i} \neq p_{i} \\ w\left(x_{i}, z_{1}\right) & \text { if } z_{1}=z_{2} \text { and } o_{i}=p_{i} .\end{cases}
$$

The $\hat{B}$ operators, on the other hand, are constructed to cause interference (constructive or destructive depending upon the output class) between the amplitudes of competing eigenstates. They increase the probability amplitudes (positively or negatively) of those eigenstates that correspond to features that match their associated input training vector regardless of output class. Finally, the pattern operators are combined as follows:

$\hat{A}$ as the operator $\sum_{i=1}^{q} \hat{A}_{x_{i} o_{i}}^{r}$,

$\hat{B}$ as the operator $\sum_{i=1}^{q} \hat{B}_{x_{i} o_{i}}^{r}$, and

$\hat{T}$ as the operator $\hat{A} \hat{B}$. 
$\hat{T}$ combines the information in $\hat{A}$ and $\hat{B}$ to represent an exponential number of patterns in linear superposition for the purpose of pattern association. The learning of the patterns in $T$ by the QuAM may be summarized as follows.

1. Prepare $|\psi\rangle=\sum_{z p}^{\mathrm{rr}} c_{z p}^{\mathrm{rr}}|z p\rangle$ such that the $c_{z p}^{\mathrm{rr}}$ represent a uniform distribution.

2. Calculate the operator $\hat{T}$.

3. Apply the operator $\hat{T}$ to $|\psi\rangle$.

In essence, the information learned from the training set is encoded in the coefficients $c_{z p}^{r r}$. For the execution or pattern completion phase two more definitions are required.

$\stackrel{r}{y}$ as a binary input vector to be classified, and

$\hat{Y}$ as an operator defined as the matrix

$$
y_{z_{1} p_{1}, \underset{z_{2}}{r} \underset{r}{r} p_{2}}^{r}= \begin{cases}0 & \text { if } z_{1} \text { does not match } y_{i} \\ w\left(y_{i}, z_{1}\right) & \text { if } \underset{z_{1}}{r}=z_{2}\end{cases}
$$

$\hat{Y}$ acts similarly to $\hat{B}$ in that it makes non-zero the probability amplitudes for those eigenstates whose features match the input vector to be classified; obviously output class cannot be considered. Given the input vector $y$, pattern completion proceeds as follows.

4. Apply the operator $\hat{Y}$ to $|\psi\rangle$.

5. Observe the quantum spin-1/2 systems associated with the output vector.

Concisely, to complete the partial pattern described by an input vector $y$ given a training set $T$, the quantum state $|\psi\rangle$ is prepared to be in a superposition of all its eigenstates, and then

$$
\hat{Y} \hat{T}|\psi\rangle
$$

is calculated. Finally, the system $|\psi\rangle$ is observed causing it to choose a single eigenstate, and the values observed for the spin-1/2 systems associated with the output pattern are the completion of $y$.

\section{Discussion and empirical results}

Three datasets from the UCI repository [9] were selected for empirical testing. Due to the exponential nature of simulating quantum systems on a classical computer, only small data sets (in the number of inputs) can be considered. These data sets were converted to binary representation before simulation. The Lenses data set represents patients and what kind of contact lense they should be prescribed (hard, soft, none). The Hayes data set is an abstract measure of concept formation used to test human psychological function. The $L E D$ data set is an artificially generated representation of the 10 digits of an LED display with $25 \%$ noise. Ten fold cross validation was used to obtain results, and since quantum mechanics is a probabilistic theory, ten trials for each data split were run to get a more accurate picture of the results.

Table 1. Pattern completion accuracy

\begin{tabular}{llllll}
\hline & $\rho=2$ & $\rho=4$ & $\rho=8$ & $\rho=16$ & $\hat{A}$ \\
\hline Lenses & 0.77 & 0.98 & 1.00 & 1.00 & 1.00 \\
Hayes & - & - & 0.90 & 0.91 & 0.91 \\
LED & - & - & 0.63 & 0.66 & 0.73 \\
\hline
\end{tabular}

For the Lenses data set, the QuAM uses 5 neurons (3state qubits) to represent 23 patterns; for the Hayes data set, the QuAM uses 8 neurons to represent 83 patterns; and for the $L E D$ data set, the QuAM uses 7 neurons to represent 200 (noisy) patterns. Table 1 shows the pattern completion rate on the data sets for different values of $\rho$ (from the $m$ function). The first four columns, labeled with their respective values for $\rho$, show that as $\rho$ increases in value, pattern recall accuracy improves. This is due to the nature of the $m$ function and the length of the input vector $x$. This improvement is most marked for the Lenses data set which achieves perfect recall of all 23 patterns using only 5 qubits. The results for the Hayes data set are equally encouraging -- $90 \%$ recall of 83 patterns using only 8 qubits. Finally notice that for the $L E D$ data set the results are not as good. This is due to the noise in the data set producing harmful interference patterns through operator $\hat{B}$. The fifth column shows recall accuracy using the $\hat{A}$ operator instead of the $\hat{T}$ operator. In this case, results for $L E D$ are also near optimum (note that due to noise the maximum accuracy for $L E D$ is $75 \%$ ).

The ability to memorize and correctly recall this many patterns with so few neurons is classically not possible. It is the quantum effects of coherence, interference and entanglement that make the exponential storage capacity possible.

\subsection{Generalization}

Although the QuAM's primary purpose is to memorize a set of patterns for completion, it does have the ability to generalize over patterns not seen during learning. However, since this is not its main purpose and since the QuAM's bias is towards the highest order matching feature, its generalization ability is expected to be limited. The definition of $\hat{T}$ includes the $\hat{B}$ operator for the purpose of utilizing interference between contradictory 
features in order to improve generalization. This is accomplished by having features that are good predictors of the output vector reinforced and by having features that are bad predictors eliminated. However, the interference in $\hat{B}$ sometimes overcompensates and becomes detrimental to generalization by actually reinforcing bad features. Thus, while the $\hat{B}$ operator provides some generalization accuracy, a new operator that better describes the proper constructive and destructive interference between patterns will improve this accuracy significantly. Table 2 supports this by giving generalization results using the operator $\hat{A}$ alone (no interference) and the full operator $\hat{T}$. Note that on the Lenses and Hayes data sets, the addition of the interference inherent in $\hat{B}$ does improve generalization as expected. However, due to the noise in the $L E D$ data set, $\hat{B}$ actually hurts generalization accuracy, just as it did recall accuracy (table 1). For reference, the third column, labeled "Other", provides average generalization accuracy on these data sets for several well known learning algorithms [13]. As expected, the generalization accuracy of the QuAM may still be improved significantly.

\section{Table 2. Generalization accuracy}

\begin{tabular}{lccc}
\hline & $\hat{A}$ & $\hat{T}$ & Other \\
\hline Lenses & 0.43 & 0.68 & 0.73 \\
Hayes & 0.45 & 0.48 & 0.66 \\
LED & 0.59 & 0.56 & 0.68 \\
\hline
\end{tabular}

\section{Concluding remarks}

A unique view of the pattern completion problem is presented that allows the proposal of a quantum associative memory with exponential storage capacity. It employs simple spin-1/2 and spin-1 quantum systems and represents training and input vectors as quantum operators. This approach demonstrates that learning techniques can be developed making use of the unique characteristics of quantum mechanics. Discussion on the physical realization of this system is beyond the scope of this paper. We acknowledge that the implementation is far from a trivial issue as investigation into the field of quantum computation will attest. By far the most difficult issue is the maintenance of quantum coherence throughout the computation. However, recently some excitement has been generated over the possible use of nuclear magnetic resonance (NMR) techniques to overcome many of these difficulties [4]. Finally, it may be noted that we have made use of only the very simplest of quantum systems and one of the very simplest of learning algorithms. Current research is focused on improving generalization capability for patterns not memorized during learning. Future work will include application of quantum theory to more complex learning systems, application of more complex quantum systems to the artificial neural network paradigm, and extension to continuously valued domains.

\section{References}

[1] Barenco, A., C. H. Bennett, R. Cleve, D. P. DiVincenzo, N. Margolus, P. Shor, T. Sleator, J. Smolin, and H. Weinfurter, "Elementary Gates for Quantum Computation", Physical Review A, vol. 52, pp. 3457-3467, 1995.

[2] Behrman, E. C., J. Niemel, J. E. Steck and S. R. Skinner, "A Quantum Dot Neural Network", IEEE Transactions on Neural Networks, submitted, 1996.

[3] Brassard, G., "New Trends in Quantum Computation", Proceedings of the 13th Annual Symposium on Theoretical Aspects of Computer Science, pp. 3-10, Grenoble, France, Springer, 1996.

[4] Cory, David G., Mark D. Price, Amr F. Fahmy and Timothy F. Havel, "Nuclear Magnetic Resonance Spectroscopy: An Experimentally Accessible Paradigm for Quantum Computing", Physica D, to appear, (accepted Aug. 27, 1997).

[5] Deutsch, D. and Richard Jozsa, "Rapid Solution of Problems by Quantum Computer", Proceedings of the Royal Society, London A, vol. 439, pp. 553-8, 1992.

[6] Deutsch, D., "Quantum Theory, the Church-Turing Principle and the Universal Quantum Computer", Proceedings of the Royal Society, London A, vol. 400, pp. 97-117, 1985.

[7] Feynman, R. P., R. B. Leighton and M. Sands, The Feynman Lectures on Physics, vol. 3, AddisonWesley Publishing Company: Reading Massachusetts, 1965.

[8] Menneer, T. and A. Narayanan, "Quantum-inspired Neural Networks", technical report R329, Department of Computer Science, University of Exeter, Exeter, United Kingdom, 1995.

[9] Mertz, C. J. and P. M. Murphy, UCI Repository of Machine Learning Databases, University of California at Irvine, Department of Information and Computer Science, http://www.ics.uci.edu/ mlearn/ MLRepository.html, 1996.

[10] Perus, M., "Neuro-Quantum Parallelism in Brain-Mind and Computers", Informatica, vol. 20, pp. 173-83, 1996.

[11] Shor, P. W. "Polynomial-Time Algorithms for Prime Factorization and Discrete Logarithms on a Quantum Computer", SIAM Journal of Computing, vol. 26 no. 5, pp. 1484-1509, 1997.

[12] Ventura, D. and T. Martinez, "An Artificial Neuron with Quantum Mechanical Properties", Proceedings of the International Conference on Artificial Neural Networks and Genetic Algorithms, to appear, 1997.

[13] Zarndt, Frederick, "A Comprehensive Case Study: An Examination of Machine Learning and Connectionist Algorithms", Masters Thesis, Brigham Young University, 1995. 